\title{
Workplace based assessment (WPBA) in dental education- A review
}

\author{
Varsha S Manekar, ${ }^{1, *}$, Sulbha A. Radke ${ }^{2}$ \\ Associate Professor, ${ }^{1}$ Dept. of Oral \& Maxillofacial Surgery, ${ }^{2}$ Dept. of Conservative Dentistry \& Endodontics, Government \\ Dental College \& Hospital, Nagpur, Maharashtra, India
}

*Corresponding Author:

Email: varsha_manekar@yahoo.co.in

\begin{abstract}
Aim: To describe work place based assessment (WPBA) and their possible application in dental education- an overview. The dental students need to develop and master the clinical procedural skills in preclinical and clinical postings. The dental education imparted to these students is from cognitive as the psychomotor domain. Going through the curriculum of the dentistry at undergraduate and post graduate level, the students have to master the complex procedural skills. Can we take their formative assessment as workplace-based assessment? The WPBA is performed in clinics with real patients. Workplace-based assessments target the highest level of the Miller's pyramid by collecting information about how doctors perform in their normal practice and how they behave in their real life on a day-to-day basis. WPBA help in evaluating students, from all aspects the cognitive, psychomotor, communication skills, management and professionalism. The formative assessment systems being used today are taken in clinics in all clinical subjects, but they are not structured. Norcini JJ in his article on work place based assessment described various assessments in working place. These include mini-CEX, CEC, CWS, BPE, DOPS, CbD, MSF $\left(360^{\circ}\right)$. Although awareness of these techniques is increasing, very few dental faculties from various dental schools have carried out the studies of these WPBA. This article is aimed at detailing the various WPBA methods available. The author has searched the literature for articles describing these techniques to concise these. These are used in medical education in few institutes as per literature. This overview of WPBA will help the dental educators to utilize these WPBA in their institutes.

Conclusion: This article reviewed the WPBA from the literature. The dentistry being a procedure based, the trainees can be benefitted from this formative assessment. The dental education in our country can implement WPBA for the competency of trainees and improved dental health care.
\end{abstract}

Keywords: Work place based assessment, DOPS, Dental education, Three sixty degree assessment.

The dental students need to develop and master the procedural skills in preclinical and clinical postings. The dental education imparted to these students is from cognitive as well as the psychomotor domain. Going through the curriculum of the dentistry at undergraduate and post graduate level, the students have to master the complex procedural skills. Can we take their formative assessment as workplace-based assessment (WPBA)? WPBA is the formative assessment in clinics with real patients. This may help in evaluating students, from all aspects the cognitive, psychomotor, communication skills, management and professionalism. The formative assessment systems being used today are taken in clinics in all clinical subjects, but they are not structured. They are not assessing for communication skills, management and professionalism. Norcini JJ (2007) in his article on WPBA described the new formative assessments for medical education.

The Millers Pyramid (1990) is the model of the development of competence. This is the most commonly cited relating to assessment. This is a conceptual model which encompasses the elements required for clinical competence - from the underpinning cognitive levels of knowledge and application of knowledge (Knows and Knows How) to the behavioural levels of practical competence, perhaps demonstrated on a model (Shows) and how a doctor (or dentist) actually performs in practice with patients (Does) (Miller, 1990) (Fig. 1). The assessment of clinical competence, using WBAs, has been based on this work by George Miller. Miller's 'Framework for Clinical Assessment' focuses on what occurs in professional practice rather than what happens in an artificial setting or test situation ${ }^{1}$ (Miller, 1990) (see Fig. 1).

Dental undergraduates as well as postgraduates have responsibility of practical delivery of complex irreversible treatment with a focused exposure to broader procedural skills. The psychomotor, and attitudinal skills are the essential components of the procedural skills along with cognitive domain. The assessment methods used mostly assess the cognitive domain. The assessment of procedural skills at the works place can be more appropriate for the dental students. The assessments at the lower level of Miller's Pyramid focus more on knowledge domain. "Does" level of Pyramid assess the students on a real patient encounter which are designed to assess the clinical skills, attitudes, and behaviours of students that are essential in providing high quality patient care. It involves direct observation of real patient encounters followed by one on one structured feedback sessions by observing faculty. ${ }^{1}$

Formative assessment is an instructional intervention evaluating performance and identifying trainees' strengths and weaknesses in order to reveal performance gaps, that is, differences between desired and actual performance. ${ }^{2-4}$ Although assessment of clinical competence is receiving increasing attention in educational research, it has not yet yielded a single 
'gold-standard' performance assessment tool that can confidently be said to be both reliable and valid. Formative assessments, for example WPBAs, encourage a self-reflective process during learning and teaching, by providing trainees with feedback on their progress towards the acquisition of knowledge, understanding and skills, and their development of professional attitudes and insights ${ }^{5}$. Norcini ${ }^{6}$ has summarized the primary weak areas of the traditional assessment method, namely, lack of assessment of a large variety of cases, examination by a very limited number of examiners and testing of only a few competencies. These all lead to a lack of reproducibility of scores.

Workplace-based assessment (WPBA) has been defined as the 'assessment of day-to-day practices undertaken in the working environment'. WPBAs are designed to assess a clinician's performance on a day-today basis in real life working conditions. Workplacebased assessments have a number of potential advantages $^{7}$ (Tooke, 2008). They offer a formative assessment tool by offering information about one's actual performance in the workplace rather than in the artificial environment of a summative examination. They complement the more traditional examination-based assessment of knowledge and thus afford a more holistic and comprehensive assessment of trainees' progress. They provide an opportunity to improve training and facilitate interaction with the trainee but they also, ultimately, aim to improve patient care. ${ }^{8}$ (Davies, 2005)

Going through the literature the application of WPBA is seen in the UK dental universities back in 2007. The other universities world-wide has used it for the formative assessment of the undergraduate dental students and specifically for dental postgraduates. If we go through our set up of dental education in India, we have formative assessment as part of curriculum in most of universities. Specifically going through the list of WPBA these are not practised. There are very few evidence in literature showing its application in dental student's education. Whereas the practical \& clinical based assessment system is existing. This system itself needs standardization according to the methods of WPBA described in literature. This article describes the WBAs which can be used in undergraduate and postgraduate dental training and explores their strengths, weaknesses, perceived value by trainees and trainers and how these tools can be used in a reliable and valid way.

There are growing numbers of WPBAs of increasing sophistication to assess different types of competencies within each of the four domains. Although the range of assessment methodologies can appear bewildering, the vast majority fall within one of three broad types. All WPBAs are in essence 'observational', with variable amounts of dialogue. The first type, in which the majority of WPBAs reside, rely normally on one evaluator, who is usually a trainer or supervisor, observing an aspect of professional practice and scoring and commenting appropriately, for example mini- clinical evaluation exercises; direct observation of procedural skills; and dental evaluation of performance and procedure-based assessments. The second type involves discussion of clinical cases seen or treated by the trainee such as case-based discussions. The third type involves obtaining feedback, usually by means of questionnaires or surveys, from a variety of sources related to the workplace, type includes the mini-peer assessment tool, team assessment of behaviour, $360^{\circ}$ assessments and multisource feedback, and all involve receiving feedback from a combination of colleagues, staff and patients. ${ }^{9}$

The various methods of WPBA can be listed as:

1. Mini-Clinical Evaluation Exercise (mini-CEX);

2. Clinical Encounter Cards (CEC);

3. Direct Observation of Procedural Skills (DOPS);

4. Blinded Patient Encounters (BPE);

5. Case-based Discussion (CbD);

6. MultiSource Feedback (MSF).

Dental evaluation of performance skill can be performed with Mini-Clinical Evaluation Exercise (mini-CEX) and Direct Observation of Procedural Skills (DOPS). Especially in specialty these procedural based training assessment is indicated. Direct observation of a trainee by one examiner during a clinical encounter with a real patient in the normal work setting e.g. on a ward or in a dental clinic.

Mini-Clinical Evaluation Exercise (mini-CEX): This is used formatively with feedback. The skills assessed are history taking, physical examination, diagnostic, clinical judgement, decision making, communication and time management. In the mini-CEX, the "snap-shot" observation lasts 15 to 20 minutes and is followed by immediate feedback from the examiner. Typically, multiple mini-CEXs are used with a variety of patients. The observation is marked using a standardised tick box form that is used to record information about the case, setting, trainee and examiner ${ }^{10}$ (Norcini). Performance is rated for a list of skills as: at, above or below expectation. Mini-CEX are primarily used formatively with feedback to produce an action plan that is structured to support the trainee's learning. With a certain amount of planning, the mini-CEX is feasible and can be fitted into routine clinical training. Its Reliability increases with the number of encounters with 6 to 8 mini-CEXs giving acceptable reliability. Assessor training is also important for reliability and to improve the quality of feedback. It has high authenticity and its reliability increases with number of examinations (mini-CEXs) performed. Originally Mini-CEX was designed by J. Norcini ${ }^{11}$ in 1995 in the USA for the evaluation of Internal Medicine residents' clinical skills.

In the original work, each aspect of the clinical encounter is scored by a faculty member using a 9 -point rating scale where $1-3$ is unsatisfactory, $4-6$ is satisfactory and $7-9$ is superior. The parameters evaluated include: interviewing skill, physical 
examination, professionalism, clinical judgement, counselling, organization and efficiency, and overall competence. This assessment tool has been shown to be a reliable way of assessing postgraduate trainee performance provided there is sufficient sampling. Roughly four encounters are sufficient to achieve a 95\% confidence interval of less than 1 (on the 9-point scale) and approximately $12-14$ are required for a reliability coefficient of $0.8^{11}$ (Norcini et al. 1995, 2003).

Mini-CEX, in contrast, has the potential to be a more practically suited assessment tool in situations involving patient-doctor interactions and where communication skills and professionalism are important. Mini-CEX formalizes the supervisory interaction between teachers and students and promotes teaching interactions. As teachers are asked to observe their students, students have to take responsibility for the case, and teachers learn more about their students' skills and decision-making processes. The structured nature of the rating form means that teachers give feedback across a broader range of topics and are more inclined to address issues that otherwise may not be addressed in evaluation. However, the assessment can be perceived as "threatening" to students and may alter how they perform, and also change the nature of the collegial relationship between the teacher and the student by strongly emphasizing the 'assessor' role of the teacher ${ }^{12}$.

Clinical encounter cards (CEC): The performance of trainees can be assessed and scored by direct observation of a patient encounter by clinical encounter cards. The CEC system has been developed at McMaster University in Canada (Hatala \& Norman 1999) ${ }^{13}$. This is similar to mini-cex. The encounter card system scores the following dimensions of observed clinical practice: history-taking, physical examination, professional behaviour, technical skill, case presentation, problem formulation (diagnosis) and problem solving (therapy). Each dimension is scored using a 6-point rating scale describing performance as 1: unsatisfactory, 2: below the expected level of student performance, 3 : at the expected level of student performance, 4 : above the expected level of student performance, 5: outstanding student performance, and 6: performance at the level of a medical graduate. In addition to capturing the quality of the performance, the 4.6 inch score cards also provide space for assessors to record the feedback given to the trainee at the end of the encounter. ${ }^{14}$

This system has been shown to be a feasible, valid, and reliable measure of clinical competence, provided that a sufficient number of encounters (approximately 8 encounters for a reliability coefficient of 0.8 or more) are collected (Hatala \& Norman 1999). ${ }^{13}$ This assessment method in the UK, focuses on evaluating the procedural skills of postgraduate trainees by observing them in the workplace setting (Wragg et al. 2003). ${ }^{15}$ Trainees' performance is scored using a 6-point rating scale where 1-2 is below the expected level of competency, 3 reflects a borderline level of competency, 4 meets the expected level of competency and 5-6 are above the expected level of competency. The assessment procedure is generally expected to require 15 minutes of observation time and 5 minutes dedicated to feedback.

Direct observation of procedural skills (DOPS): This assessment skill focuses on evaluating procedural skill of students. Although DOPS is similar to procedural skills log books, the purpose and nature of these methods differ significantly. The recording of procedures is common to both of them, but log books are usually designed to ensure that trainees have simply performed the minimum number required to be considered competent. The provision of structured feedback based on observation of a performance is not necessarily part of the log book process. Moreover, the procedure is not necessarily performed under direct observation and little feedback, if any, is expected to be given. In contrast, DOPS ensures that trainees are given specific feedback based on direct observation so as to improve their procedural skills. ${ }^{14}$

DOPS is designed specifically to assess practical skills in a workplace setting. A student is observed and scored by an assessor while performing a routine practical procedure during his / her normal clinical work. The assessor uses a standard DOPS form to score the technique. The student is deemed either competent or incompetent. For any particular skill the student usually has to pass a number of repeated assessments (typically six) to be signed off as competent at that skill. Alternatively, the student may also request this type of assessment when they have judged that they have developed the required level of competency. Skills Assessed are Practical / technical ability and manual dexterity in a work place setting. DOPS are run during normal clinical work and, with a certain amount of planning and organisation, this represents a feasible way of assessing the key procedures and practical skills required for particular disciplines / specialties. Key points of DOPS are: High authenticity; Multiple assessments of the same skill; Present a valuable opportunity for formative feedback with written marking sheet; Criterion referenced marking; Emphasis upon testing psychomotor skills; Resource intensive to conduct the assessment and need suitable cases ${ }^{16 .}$

DOPS is a method of assessment developed specifically for assessing practical skills. It requires an educational supervisor to directly observe the trainee performing a certain procedure, make judgments about specific components of the procedure, and grade the trainee's performance. However, the high variation between individual assessors can often influence the efficacy of the results unless rigorous standardization of assessors is undertaken. ${ }^{17}$ The procedures performed by undergraduates such as cavity cutting, manipulation of materials, local anaesthesia techniques, extraction of teeth, scaling, impression taking, from various clinical subjects can be formatively assessed and the feedback 
can improve the student's skill. Whereas the post graduate trainees are performing complex technique sensitive procedures, DOPS can specifically of benefit for them.

Blinded patient encounters: Students, in groups of 4 5 , participate in a bedside tutorial. It starts with a period of direct observation in which one of the students in the group is observed performing a focused interview or physical examination as instructed by the clinician educator conducting the teaching session. Thereafter the student is expected to provide a diagnosis, including a differential diagnosis, based on the clinical findings. The patient is unknown to the student, hence the term 'blinded' patient encounter (McLeod \& Meagher 2001). ${ }^{18}$

This type of patient encounter has the advantage of safely allowing the trainee to practice information gathering, hypothesis generation, and problem solving without access to the workup by more senior doctors. After the presentation, the session focuses on demonstrating the important clinical features of the case as well as discussing various issues, for example appropriate investigation and treatment relevant to the patient's presenting clinical problem. It concludes with a feedback session in which the student receives personal private advice about his/her performance. Feedback is provided using a 9-point rating scale for assessment of clinical interviewing and examination skills as well as clinical reasoning skills. The rating scale ranges from $1-$ 3 for poor performance, 4-6 for adequate performance and 7-9 for good performance. Space is provided on the score sheet to add other written comments. Students keep the score sheets which are only used for feedback purposes. $^{14}$

Case-based discussion (CbD): This assessment method is an anglicised version of ChartStimulated Recall (CSR) developed for use by the American Board of Emergency Medicine (Maatsch et al. 1983). ${ }^{19}$ Discussion of clinical cases: These are semi-structured discussions known as case-based discussions (CbDs) regarding the management of a patient treated or seen by the trainee, for example during a consultation clinic. The trainee is responsible for selecting the cases, but the trainee and evaluator should ensure that cases are well balanced and representative. The trainee usually presents at least two cases with the relevant patient records, radiographs and study casts, to the evaluator before the discussion is due to take place. The evaluator then selects one of these cases for discussion and judges the trainee's performance across several broad criteria such as clinical judgement, treatment planning and decision making skills. CbDs evaluate the trainee's understanding and rationale for the treatment provided. The evaluator should ensure that as many competencies are covered as possible for each case selected. ${ }^{20}$
Multi Source Feedback (MSF): More commonly referred to as 360-degree assessment, this method represents a systematic collection of performance data and feedback for an individual trainee, using structured questionnaires completed by a number of stakeholders. The assessments are all based on directly observed behaviour (Wragg et al. 2003) but they differ from the methods presented above in that they reflect routine performance, rather than performance during a specific patient encounter. ${ }^{15}$

Although there are a number of different ways of conducting this form of assessment, the mini-peer assessment tool (miniPAT) that has been selected for use in the Foundation Programme in the UK is a good example. Trainees nominate 8 assessors including senior consultants, junior specialists, nurses and allied health service professionals. Each of the nominated assessors receives a structured questionnaire which is completed and returned to a central location for processing. Trainees also complete self-assessments, using the same questionnaires, and submit these for processing. The categories of assessment include: good clinical care, maintaining good clinical practice, teaching and training, relationships with patients, working with colleagues and an overall assessment. The questionnaires are collated and individual feedback is prepared for trainees. Data are provided in a graphic form which depicts the mean ratings of the assessors and the national mean rating. All comments are included verbatim, but they remain anonymous. Trainees review this feedback with their supervisor and together work on developing an action plan. This process is repeated twice yearly during the training period. This method is widely used in industry and business, but has also been found to be useful in medicine. $^{15}$

\section{Feedback}

Feedback is an important component of WPBA. According to Shepard, ${ }^{21}$ feedback promotes student learning in three ways: 1) it informs trainees of their progress or lack thereof; 2) it advises trainees regarding observed learning needs and resources available to facilitate their learning; and 3) it motivates trainees to engage in appropriate learning activities.

Providing feedback that focuses on the process can be of more value because it encourages a deeper appreciation of the performance. This involves giving feedback that enhances an understanding of relationships (the construction of meaning), cognitive processes, and transfer to different or novel situations (Marton et al. 1993)..$^{22}$

\section{Discussion}

The growing number of WPBAs can be misunderstood by trainees and trainers, in particular how to use them in a reliable and valid way. Their strengths, weaknesses, perceived value by trainees and trainers have been explored to provide guidance to those 
embarking on the journey of using WPBAs in assessment of dental students.

Prescott-Clements $^{5}$ LE et al mentioned that formative assessments, for example WPBAs, encourage a self-reflective process during learning and teaching, by providing trainees with feedback on their progress towards the acquisition of knowledge, understanding and skills, and their development of professional attitudes and insights. The feedback received from trainees in few studies are- WPBAs can provide support, increase confidence and highlight areas of weakness for development. Scoring is usually perceived as fair and an accurate representation of ability. Trainers perceive WPBAs to be clear, comprehensive, reflective and to highlight trainees with difficulties. ${ }^{23}$ However, assessments may be seen as time consuming with considerable paperwork or as having an adverse impact on training. ${ }^{24}$

Following feedback, the willingness of trainees to change their behaviour is variable and can depend upon the manner with which the feedback is delivered and the support offered to effect change. ${ }^{25}$ The time of the assessment, the training of assessors (trainers) as well as trainees, the positiveness of feedback are few variables discussed in the literature on WPBA. It is argued that with WPBAs there exists a strong subjective element as to whether any given competency is achieved. It has been pointed out that a competency-based approach leads trainees on a superficial path towards achieving a set of discrete and narrow prescribed skills, with little attention paid to the relationships associating the individual competencies and the deeper meaning underlying each task (Leung, 2002). ${ }^{26}$

WPBAs are an essential tool that complements assessment methodologies such as examination to ensure that trainees develop the required proficiencies in a supervised environment. No other assessment tool reflects the competency to 'do' and this forms the underlying importance of WPBAs. However, no research has reported the beneficial or negative outcomes of WPBAs. Regular assessment with multiple assessors, unique scenarios at each assessment and providing immediate feedback can maximise the benefits of WBAs. ${ }^{20}$

\section{Conclusion}

This article reviewed the WPBA from the literature. The dentistry being a procedure based, the trainees can be benefitted from this formative assessment. The dental education in our country can implement WPBA for the competency of trainees and improved dental health care. There is need of educational research on WPBA to confirm its merits \& demerits, its feasibility and acceptance by trainees as well as trainers. There is scope for research on outcomes such as learning, implementation of clinical skills, improved patient care along with formative performance assessment. The literature is lacking in how, why \& when to actually use
WPBA and their associated problems. Also, its comparison to existing formative assessment. In conclusion WPBA is a strong tool if used properly definitely result in improvement of the dental trainee's competency.

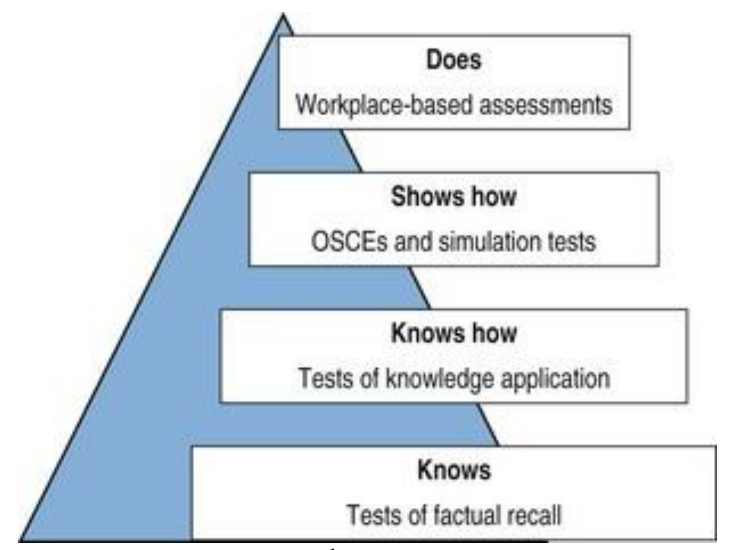

Fig 1: Millar's pyramid ${ }^{1}$

\section{References}

1. Miller GE. The assessment of clinical skills /competence /performance. Acad Med 1990:s63-7.

2. Black P, William D. Assessment and classroom learning. Assess Educ 1998;5:7-74.

3. Krasne S, Wimmers PF, Relan A, Drake TA. Differential effects of two types of formative assessment in predicting performance of first-year medical students. Adv Health Sci Educ Theory Pract 2006;11:155-71.

4. Rudolph JW, Simon R, Raemer DB, Eppich WJ. Debriefing as formative assessment: Closing performance gaps in medical education. Acad Emerg Med 2008;15:1010-6.

5. Prescott-Clements L E, van der Vleuten C P, Schuwirth L, Gibb E, Hurst Y, Rennie J S. Measuring the development of insight by dental health professionals in training using workplace-based assessment. Eur J Dent Educ 2011;15:159-64.

6. Norcini JJ. Death of the long case? BMJ 2002;324:408-9

7. Tooke J. Aspiring To Excellence: Findings and Recommendations of the Independent Inquiry into Modernising Medical Careers. London: Aldridge Press, 2008.

8. Davies H, Archer J, Heard S. 2005. Assessment tools for Foundation Programmes - a practical guide. BMJ Career Focus 330(74840):195-96.

9. H. K. Kalsi, J. S. Kalsi and N. L. Fisher An explanation of workplace-based assessments in postgraduate dental training and a review of the current literature $\mathrm{Br}$ Dent $\mathrm{J}$ 215(10):519.

10. Norcini JJ, Blank LL, Duffy FD, Fortna GS. The MiniCEX: A method for assessing clinical skills. Ann Intern Med 2003;138:476-81.

11. John J. Norcini, Linda L. Blank, Gerald K. Arnold and Harry R. Kimball, The Mini-CEX (Clinical Evaluation Exercise): A Preliminary Investigation. Ann Intern Med 1995:123:795-799.

12. Weller JM, Jones A, Merry AF, Jolly B, Saunders D. Investigation of trainee and specialist reactions to the mini-Clinical Evaluation Exercise in anaesthesia: Implications for implementation. Br J Anaesth 2009; 103:524-30. 
13. Hatala R, Norman GR. 1999. In-training evaluation during an Internal Medicine clerkship. Acad Med 74:S118-S120.

14. Norcini JJ. 2007. Workplace-based assessment in clinical training, in: Swanwick T. (Ed.) Understanding Medical Education series (Edinburgh, UK: Association for the Study of Medical Education).

15. Wragg A, Wade W, Fuller G, Cowan G, Mills P. 2003. Assessing the performance of specialist registrars. Clin Med 3:131-134.

16. Julie Williams et al A Guide to Assessment in Dental Education November 2015 Version 1;38.

17. Andersen RM, Davidson PL, Atchison KA, Hewlett E, Freed JR, et al. Pipeline, profession, and practice program: evaluating change in dental education. J Dent Educ 2005;69(2):239-48.

18. Gerzina TM, Mclean T, Fairley G. Dental clinical teaching: perceptions of students and teachers. J Dent Educ 2005;69(12):1377-84.

19. McLeod PJ, Meagher TW. 2001. Educational benefits of blinding students to information acquired and management plans generated by other physicians. Med Teach 23:83-85.

20. Maatsch JL, Huang R, Downing S, Barker B. 1983. Predictive validity of medical specialist examinations.
Final report for Grant HS 02038-04, National Center of Health Services Research. Office of Medical Education

21. H. K. Kalsi, J. S. Kalsi and N. L. Fisher An explanation of workplace-based assessments in postgraduate dental training and a review of the current literature. Br Dent $\mathrm{J}$ 2013;215(10):519.

22. Shepard LA. 2000. The role of assessment in a learning culture. Educ Res 29:4-14.

23. Marton F, Dall'Alba G, Beaty E. 1993. Conceptions of learning. Int J Educ Res 19:277-300.

24. Grieveson B, Kirton J A, Palmer N, Balmer M C.

Evaluation of workplace based assessment tools in dental foundation training. Br Dent J 2011; 211: E8. Wilkinson J R, Crossley J G, Wragg A, Mills P, Cowan G, Wade W. Implementing workplace-based assessment across the medical specialties in the United Kingdom. Med Educ 2008; 42:364-73.

25. Pereira E A, Dean B J. British surgeons' experiences of mandatory online workplace-based assessment. $J$ Royal Soc Med 2009;102:287-93.

26. Miller A, Archer J. Impact of workplace based assessment on doctors' education and performance: a systematic review. BMJ 2010;341:c5064.

27. Leung WC. Competency based medical training: review. BMJ 2002;325:693-96. 